\title{
Article \\ PbS Quantum Dots Saturable Absorber for Dual-Wavelength Solitons Generation
}

\author{
Ling Yun ${ }^{1}$ and Wei Zhao ${ }^{2, *}$ \\ 1 Advanced Photonic Technology Lab, Nanjing University of Posts and Telecommunications, Nanjing 210046, \\ China; yunling@njupt.edu.cn \\ 2 State Key Laboratory of Transient Optics and Photonics, Xi'an Institute of Optics and Precision Mechanics, \\ Chinese Academy of Sciences, Xi'an 710119, China \\ * Correspondence: weiz@opt.ac.cn
}

Citation: Yun, L.; Zhao, W. PbS Quantum Dots Saturable Absorber for Dual-Wavelength Solitons Generation. Nanomaterials 2021, 11, 2561. https://doi.org/10.3390/ nano11102561

Academic Editors: John Vakros, George Avgouropoulos and Efrat Lifshitz

Received: 16 July 2021

Accepted: 25 September 2021

Published: 29 September 2021

Publisher's Note: MDPI stays neutral with regard to jurisdictional claims in published maps and institutional affiliations.

Copyright: (C) 2021 by the authors Licensee MDPI, Basel, Switzerland. This article is an open access article distributed under the terms and conditions of the Creative Commons Attribution (CC BY) license (https:/ / creativecommons.org/licenses/by/ $4.0 /)$.

\begin{abstract}
PbS}$ quantum dots (QDs), a representative zero-dimensional material, have attracted great interest due to their unique optical, electronic, and chemical characteristics. Compared to oneand two-dimensional materials, PbS QDs possess strong absorption and an adjustable bandgap, which are particularly fascinating in near-infrared applications. Here, fiber-based PbS QDs as a saturable absorber (SA) are studied for dual-wavelength ultrafast pulses generation for the first time to our knowledge. By introducing PbS QDs SA into an erbium-doped fiber laser, the laser can simultaneously generate dual-wavelength conventional solitons with central wavelengths of 1532 and $1559 \mathrm{~nm}$ and $3 \mathrm{~dB}$ bandwidths of 2.8 and $2.5 \mathrm{~nm}$, respectively. The results show that PbS QDs as broadband SAs have potential application prospects for the generation of ultrafast lasers.
\end{abstract}

Keywords: fiber laser; mode locking; PbS quantum dots

\section{Introduction}

Low-dimensional materials have attracted extensive interest in applied physics due to their excellent optical, electronic, and chemical characteristics [1-3]. Two-dimensional (2D) graphene [4,5], black phosphorus [6], MXenes [7], antimonene [8], transition metal dichalcogenides [9], topological insulators [10], and one-dimensional (1D) carbon nanotubes [11,12] have been employed as saturable absorbers (SAs) to obtain ultrafast pulses in passively mode-locked fiber lasers. Among these SAs, the main problem is that it is difficult to achieve short carrier lifetime, high thermal damage, large modulation depth, and wide bandwidth in an individual material at the same time. Therefore, one of the ways to solve the problem is to find a new saturable absorption material that can effectively adjust the above parameters.

Semiconductor quantum dots (QDs) are particularly charming materials showing strong quantum confinement effects, as the size of QDs is close to the Bohr radius of the exciton [13]. Strong confinement not only produces interesting new effects but also strengthens the nonlinear optical characteristics [14]. Among numerous semiconductor QDs, PbS QDs possess smaller carrier effective masses and larger optical dielectric constant, leading to a large exciton Bohr radius ( 18 $\mathrm{nm}$ ), which makes relatively large QDs have strong quantum confinement effects [14]. Therefore, combined with the small energy bandgap $(\sim 0.41 \mathrm{eV})$ of $\mathrm{PbS}$, the wavelength range of the excitonic absorption peak is from the visible to $3 \mu \mathrm{m}$ via changing the size of PbS QDs [15]. This means that PbS QDs can promote the saturable absorption in a large spectral range by changing the size of QDs [16]. As a result, PbS QDs as SAs have been used for Q-switching or mode locking in various near-infrared lasers $[13,16,17]$. The most obvious advantages of PbS QDs-based SA are adjustable absorption peak, large third-order nonlinear susceptibility, fast response time, large modulation depth, and high damage threshold [18,19]. 
On the other hand, multi-wavelength passively mode-locked fiber lasers have been investigated extensively in the advancement of fascinating applications as optical fiber sensing, biomedical research, and wavelength division multiplexing (WDM) optical communication $[20,21]$. Several types of the saturable absorption materials that can realize multi-wavelength passive mode locking have been studied in depth [22-26]. Based on SESAM, Wu et al. realized the dual-wavelength (1553 and $1562 \mathrm{~nm}$ ) dissipative solitons in Er-doped fiber laser operating at normal dispersion regime [22]. By virtue of carbon nanotube SA, dual-wavelength vector solitons centered at 1533 and $1557 \mathrm{~nm}$ were achieved by Zhao et al. [23]. Tunable dual- and triple-wavelength dissipative solitons were obtained from a $\mathrm{Yb}$-doped fiber laser using graphene-oxide mode locker [24]. In a previous paper, we also reported the generation of a dual-wavelength polarization-locked vector solitons fiber laser using black phosphorus SA [25]. However, as far as we know, there is no report of multi-wavelength solitons operating in fiber-based PbS QDs mode-locked fiber lasers.

In this context, $\mathrm{PbS}$ QDs are fabricated via a modified hot-injection method. A dualwavelength passively mode-locked Er-doped fiber laser is realized by using fiber-based $\mathrm{PbS}$ QDs as intracavity mode-locked devices. The stable dual-wavelength conventional solitons with central wavelengths of 1532 and $1559 \mathrm{~nm}$, and $3 \mathrm{~dB}$ spectral bandwidths of 2.8 and $2.5 \mathrm{~nm}$ are obtained. By finely tuning the pump intensity and polarization state, the dual-wavelength mode locking can be switched into single-wavelength operation state.

\section{Materials and Methods}

The PbS QDs coated with oleic acid were prepared by a modified hot-injection meth-od by precisely controlling the mass of the precursor, reaction temperature, environment, and reaction time [18,27]. The preparation details are given in Figure 1a. Firstly, the $\mathrm{Pb}$ precursor was formed by putting lead oxide (Sinopharm Chemical Reagent Co., Ltd., Shanghai, China) (450 mg), octadecene (Sinopharm Chemical Reagent Co., Ltd., Shanghai, China) (30 mL), and oleic acid (Sinopharm Chemical Reagent Co., Ltd., Shanghai, China) (10 mL) into a three-necked flask and heating it at $120^{\circ} \mathrm{C}$ for $1 \mathrm{~h}$ in vacuum. Secondly, the S precursor was prepared by mixing sulfur powder (Sinopharm Chemical Reagent Co., Ltd., Shanghai, China) (32 mg) with oleic amine (Sinopharm Chemical Reagent Co., Ltd., Shanghai, China) $(10 \mathrm{~mL})$ and heated to $120^{\circ} \mathrm{C}$ until it was completely dissolved. Thirdly, the prepared S precursor was rapidly injected into a three-necked flask containing a $\mathrm{Pb}$ source under the protection of argon gas; then, it was cooled to room temperature with an ice water bath quickly. Finally, the sample was separated with ethanol and centrifuged at 12,000 r/min for $3 \mathrm{~min}$. The obtained PbS QDs were dried in vacuum, dissolved in cyclohexane solution (Sinopharm Chemical Reagent Co., Ltd., Shanghai, China), and stored at a concentration of $10 \mathrm{mg} / \mathrm{mL}$. The transmission electron microscope (TEM) (Hitachi, Tokyo, Japan) image of $\mathrm{PbS}$ QDs is given in Figure 1b, which shows that PbS QDs are spherical and have good dispersivity, and the average particle size is $\sim 5.7 \mathrm{~nm}$.

The fiber-based PbS QDs mode-locked device was prepared by dropping PbS QDs solution on the end face of an optical fiber connector and evaporated slowly at room temperature and pressure. Compared with the SA prepared by other methods such as mechanical exfoliation or CVD growth, the PbS QDs SA prepared by this scheme avoids the complicated and time-consuming growth and post transfer processes, and it not only overcomes the mechanical damage but also improves the damage threshold of the laser. Based on the dual-path detection system $[9,18]$, the nonlinear optical absorption characteristics of the PbS QDs have been studied. As illustrated in Figure 2, PbS QDs show strong saturable absorption behavior at $1550 \mathrm{~nm}$. The experimental results show that the unsaturable loss, saturation intensity, and modulation depth of the PbS QDs SA are $21 \%$, $\sim 0.22 \mathrm{MW} / \mathrm{cm}^{2}$, and $\sim 44 \%$ respectively. To the best of our knowledge, our SA exhibits high modulation depth compared with that reported to date. The corresponding digital photograph of the PbS QDs mode-locker is shown in the inset of Figure 2. 


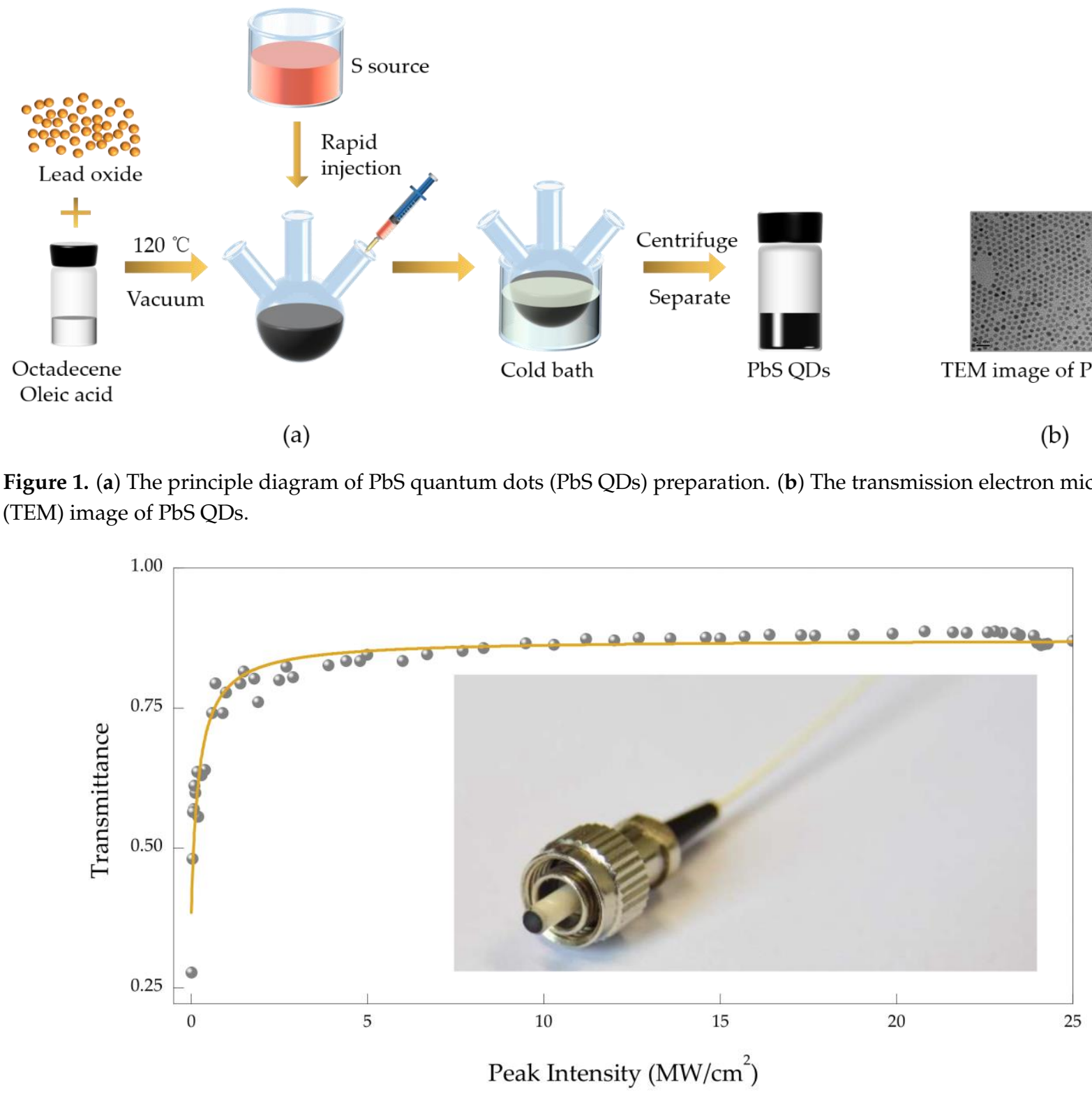

Figure 2. Nonlinear saturable absorption curve of PbS QDs saturable absorber (SA). Inset: PbS QDs mode locker.

\section{Results and Discussion}

The PbS QDs mode-locked Er-doped fiber laser operating in net anomalous dispersion regime is depicted in Figure 3. The ring cavity is composed of a $5.3 \mathrm{~m}$ erbium-doped fiber (EDF, $D=-16 \mathrm{ps} / \mathrm{nm} / \mathrm{km}$ ) and $23.2 \mathrm{~m}$ single-mode fiber (SMF, $D=17 \mathrm{ps} / \mathrm{nm} / \mathrm{km}$ ). The net cavity dispersion is about $-0.39 \mathrm{ps}^{2}$. The EDF served as a gain medium is pumped by a laser diode (LD, $980 \mathrm{~nm}$ ) (Connet, Shanghai, China) through a WDM (980/1550 nm) (Connet, Shanghai, China). A polarization-insensitive isolator (PI-ISO) provides unidirectional operation. A polarization controller (PC) (Connet, Shanghai, China) is used to adjust the polarization and balance the gain distribution of the EDF by controlling the cavity loss. The pulses are extracted from the cavity with a 10\% output coupler (OC). The PbS QDs SA device is assembled by sandwiching a fiber connector between two FC/PC fiber ferrules. The performance of the laser is recorded by an optical spectrum analyzer (Yokogawa AQ6370D) (Yokogawa, Tokyo, Japan), a second harmonic generation intensity autocorrelator (APE PulseCheck SM1600) (APE, Munich, Germany), an oscilloscope (RIGOL DS4050) (Tektronix, Johnston, OH, USA), and a radio-frequency analyzer (RS-FSV30) (Tektronix, Johnston, OH, USA). 


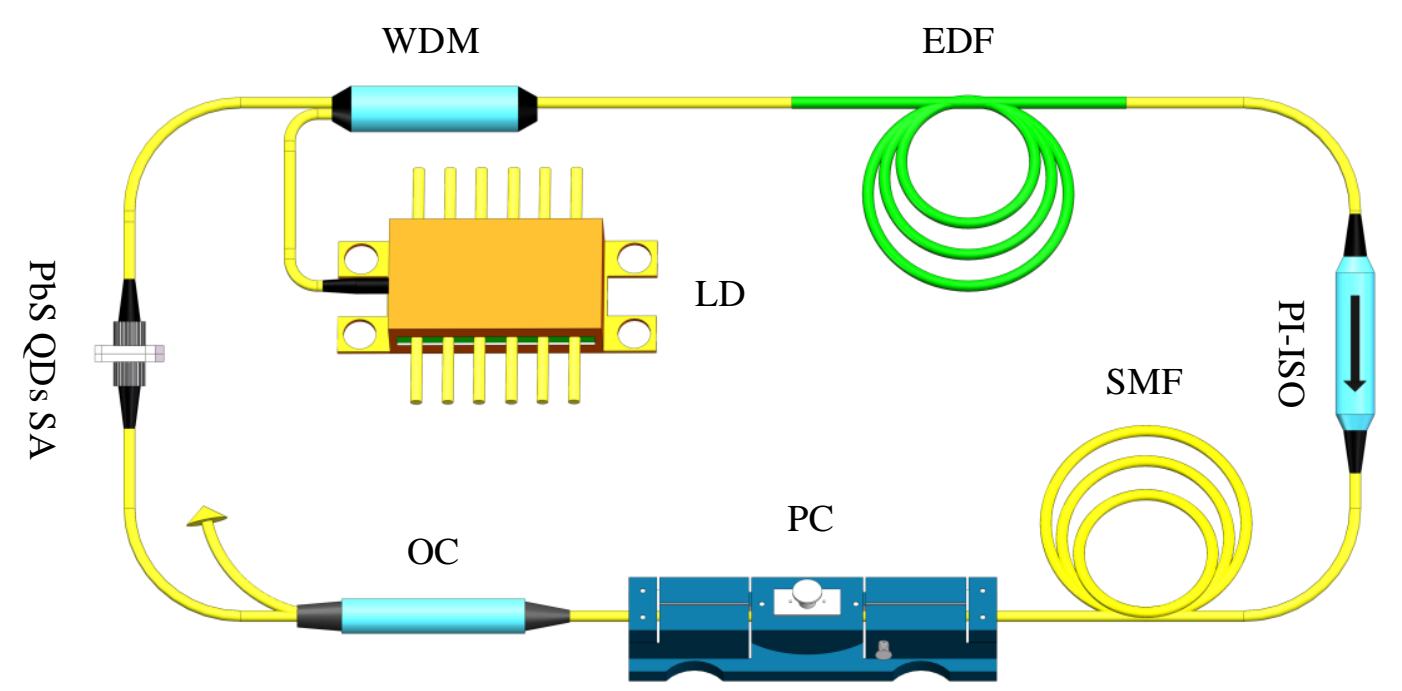

Figure 3. Laser setup. Laser diode (LD), wavelength division multiplexing (WDM), erbium-doped fiber (EDF), polarizationinsensitive isolator (PI-ISO), single-mode fiber (SMF), polarization controller (PC), output coupler (OC), and Lead sulfide quantum dots saturable absorber (PbS QDs SA).

Based on the above experimental setup, the stable dual-wavelength mode-locked laser pulses are generated when the pump strength is scaled to $200 \mathrm{~mW}$ and the PC is finely tuned, as illustrated in Figure 4. The spectrum in Figure 4a appears to be obvious Kelly sidebands, which is a typical feature of the conventional solitons in the anomalous dispersion region [28]. The central wavelengths are 1532 and $1559 \mathrm{~nm}$, and the corresponding $3 \mathrm{~dB}$ bandwidths are measured to be 2.8 and $2.5 \mathrm{~nm}$, respectively. The spectrum of dual-wavelength solitons exhibits almost the same peak intensity, and the center wavelength spacing $\Delta \lambda$ is $27 \mathrm{~nm}$. Figure $4 \mathrm{~b}$ illustrates the oscilloscope trace, in which two pulse trains are formed simultaneously. There are two conventional solitons propagating in the laser cavity, and the pulse energy of each soliton varies slightly with the height. Under proper triggering, one pulse sequence stops, and the other moves on the oscilloscope screen. The results show that the two pulse sequences have different group velocities [24]. The corresponding radio-frequency spectrum is demonstrated in Figure 4c. Different from the single-wavelength soliton mode-locked, there are two fundamental frequencies that appear in the dual-wavelength mode-locked spectrum, corresponding to two mode-locked states. The fundamental frequencies are $\sim 7.200695 \mathrm{MHz}$ and $\sim 7.201131 \mathrm{MHz}$, which are consistent with the mode-locked wavelengths of 1559 and $1532 \mathrm{~nm}$, respectively. The formation of dual-wavelength conventional solitons may be due to the interaction of EDF gain spectrum and cavity-filtering effect [29]. Both of the signal-to-noise ratios are as high as $60 \mathrm{~dB}$, which indicates good temporal stability of the PbS QDs-SA based dual-wavelength mode-locking operation. The frequency interval $\Delta f$ is $436 \mathrm{~Hz}$. Furthermore, the relationship between $\Delta f$ and $\Delta \lambda$ is theoretically expressed as [30]:

$$
\Delta f=\frac{c^{2} D \Delta \lambda}{n^{2}(L+L D \Delta \lambda c / n)}
$$

where $L$ is the fiber length, $n$ is the refractive index of fiber, $D$ is the dispersion parameter, and $c$ is the speed of light. Here, $L=28.5 \mathrm{~m}, n=1.46, D_{S M F+E D F}=10.86 \mathrm{ps} / \mathrm{nm} / \mathrm{km}$, and $c=3 \times 10^{8} \mathrm{~m} / \mathrm{s}$. Therefore, the calculated $\Delta f=434 \mathrm{~Hz}$, which is basically consistent with the experimental results. 

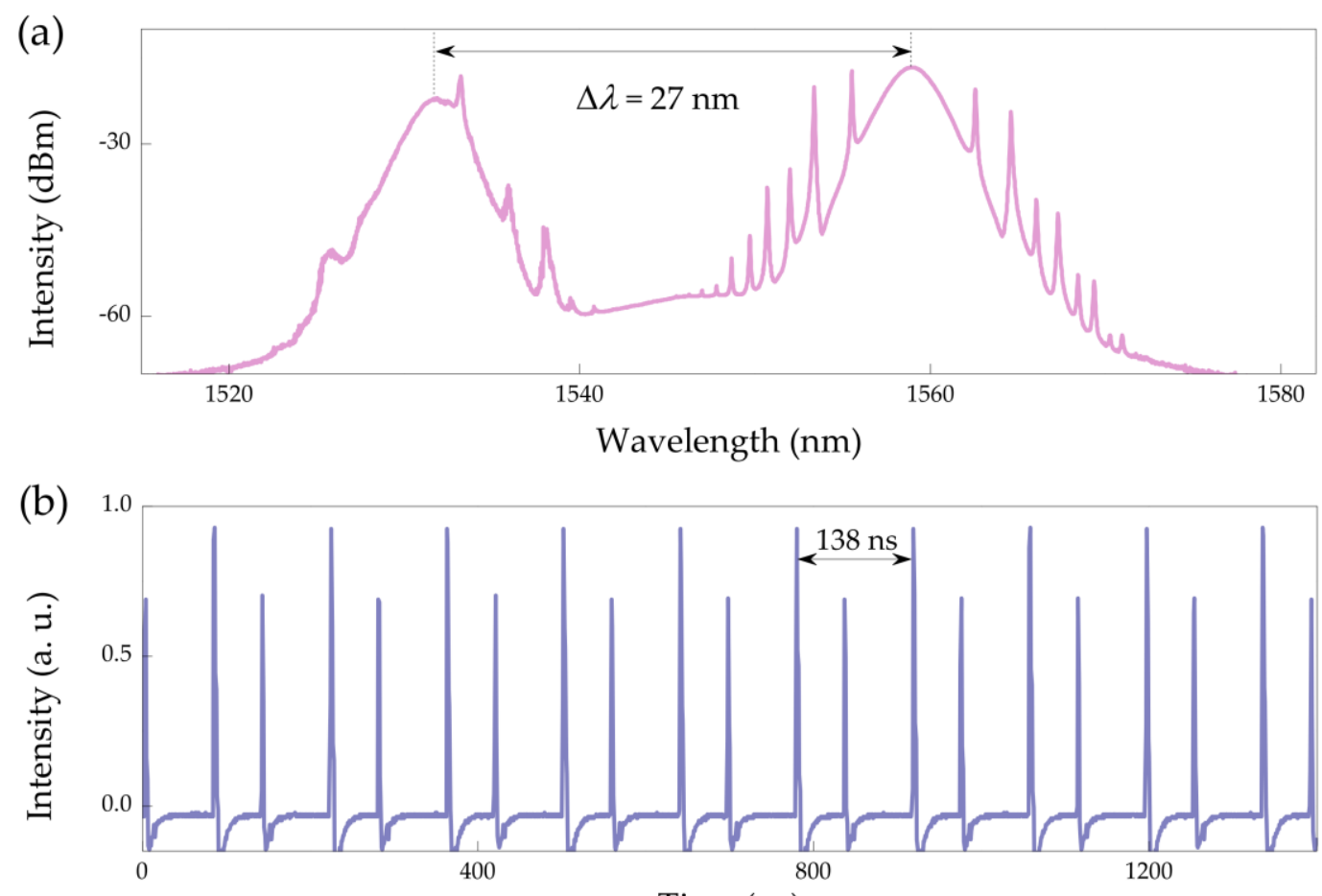

Time (ns)

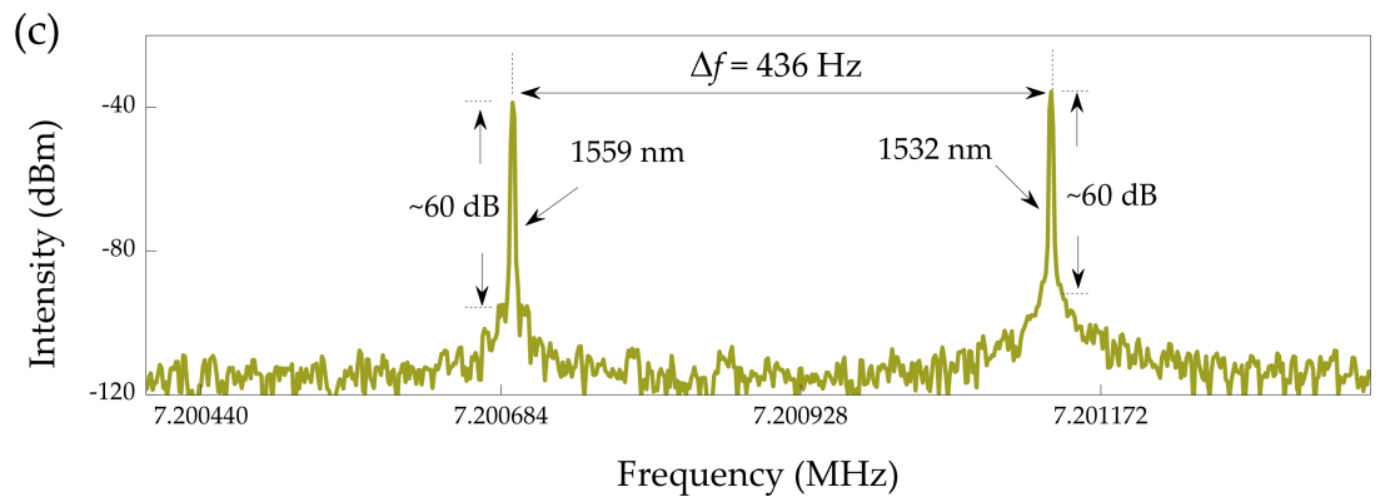

Figure 4. Dual-wavelength conventional solitons. (a) Optical spectrum at 1532 and $1559 \mathrm{~nm}$, (b) pulse train, and (c) corresponding fundamental radio-frequency spectrum.

By decreasing the pump strength to $80 \mathrm{~mW}$ and carefully tuning the PC, dualwavelength mode locking can be switched into single-wavelength mode locking. The characteristics of the proposed single-wavelength operation are presented in Figure 5. As shown in Figure 5a, the dual-wavelength conventional solitons at $1532 \mathrm{~nm}$ disappears, and the single-wavelength soliton remains at $1559 \mathrm{~nm}$. The spectrum exhibits symmetric sid bands, and the $3 \mathrm{~dB}$ bandwidth is $2.4 \mathrm{~nm}$. The autocorrelation trace is shown in Figure $5 \mathrm{~b}$. The pulse envelope is fitted with a Sech ${ }^{2}$ function. The pulse duration is $\sim 1.11 \mathrm{ps}$, so that the time-bandwidth product equals to 0.33 . Therefore, the single-wavelength conventional soliton is nearly chirp-free. Figure 5c shows the fundamental frequency of $\sim 7.200698 \mathrm{MHz}$, which is corresponding to a pulse interval of $\sim 138$ ns. The signal-to-noise ratio of the soliton is $\sim 60 \mathrm{~dB}$, indicating a stable single-wavelength mode locking. The average output power of the single pulse is $1.9 \mathrm{~mW}$, corresponding to a pulse energy and peak power of $\sim 0.26 \mathrm{~nJ}$ and $234 \mathrm{~W}$, respectively. 

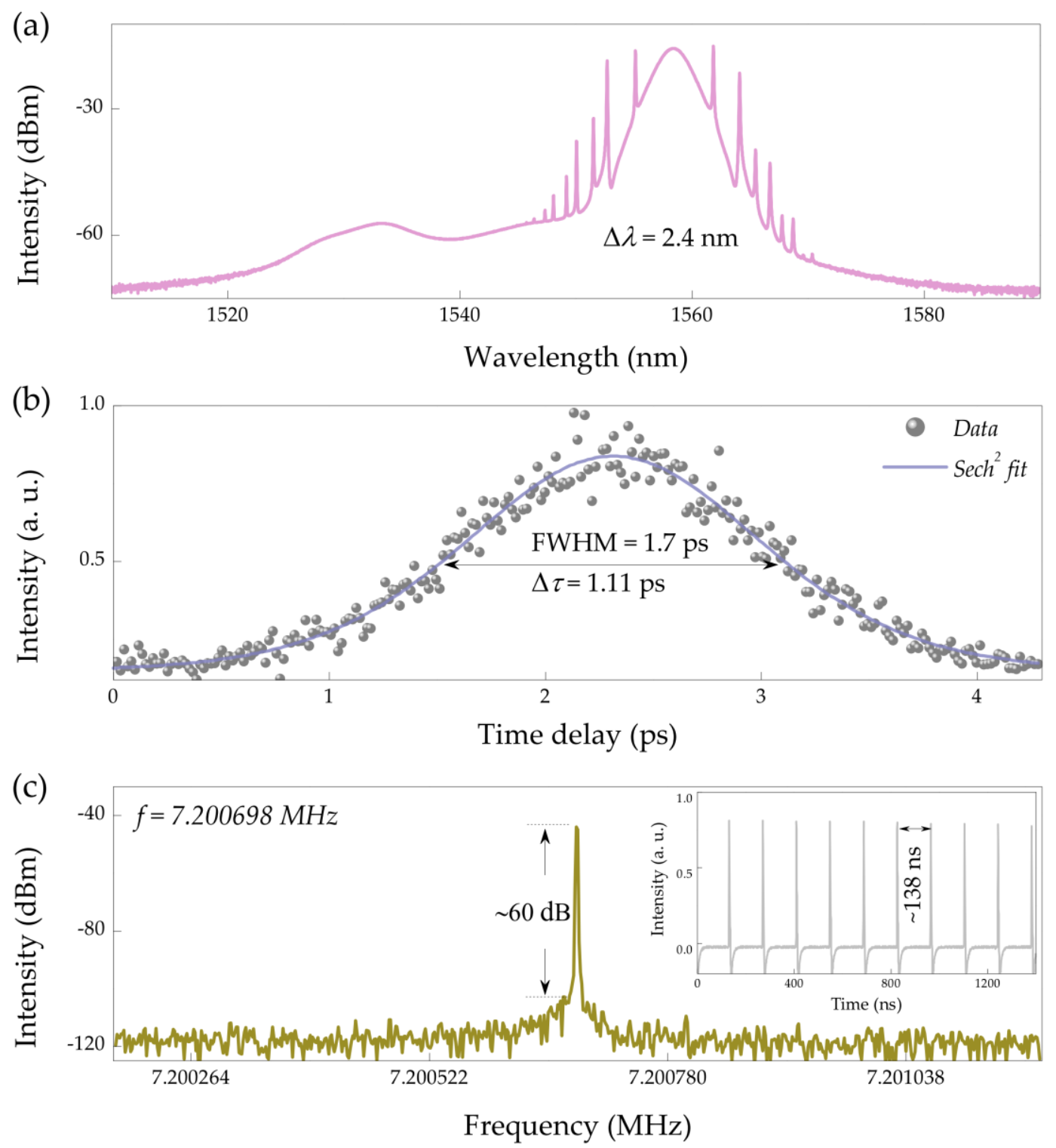

Figure 5. Single-wavelength conventional soliton. (a) Optical spectrum at $1559 \mathrm{~nm}$, (b) autocorrelation trace, and (c) corresponding fundamental radio-frequency spectrum. Inset: pulse train.

When the pump strength increases from 80 to $120 \mathrm{~mW}$, the stable single wavelength mode locking centered at $1532 \mathrm{~nm}$ with a $3 \mathrm{~dB}$ bandwidth of $3.5 \mathrm{~nm}$ is realized, as demonstrated in Figure 6. By Sech ${ }^{2}$ fitting, the pulse duration of the conventional soliton is about $0.78 \mathrm{ps}$, the corresponding time bandwidth product is calculated to be 0.36 , with slight chirp. The fundamental frequency is $\sim 7.201135 \mathrm{MHz}$, which corresponds to the round-trip time of the cavity length of the fiber laser. The radio-frequency spectrum gives a signal-tonoise ratio of $\sim 65 \mathrm{~dB}$, indicating low-amplitude fluctuations and stable single-wavelength mode-locking state. When the pump power is $500 \mathrm{~mW}$ (maximum pump power available of LD in the experiment), the mode-locking operation of the fiber laser is still stable, which shows that the fiber-based $\mathrm{PbS}$ QDs SA fiber has good thermal damage. The average output power of the laser cavity is $12 \mathrm{~mW}$, and the corresponding single pulse energy is $1.7 \mathrm{~nJ}$. Therefore, the thermal damage threshold of $\mathrm{PbS}$ QDs SA is greater than $30 \mathrm{~mJ} / \mathrm{cm}^{2}$. 

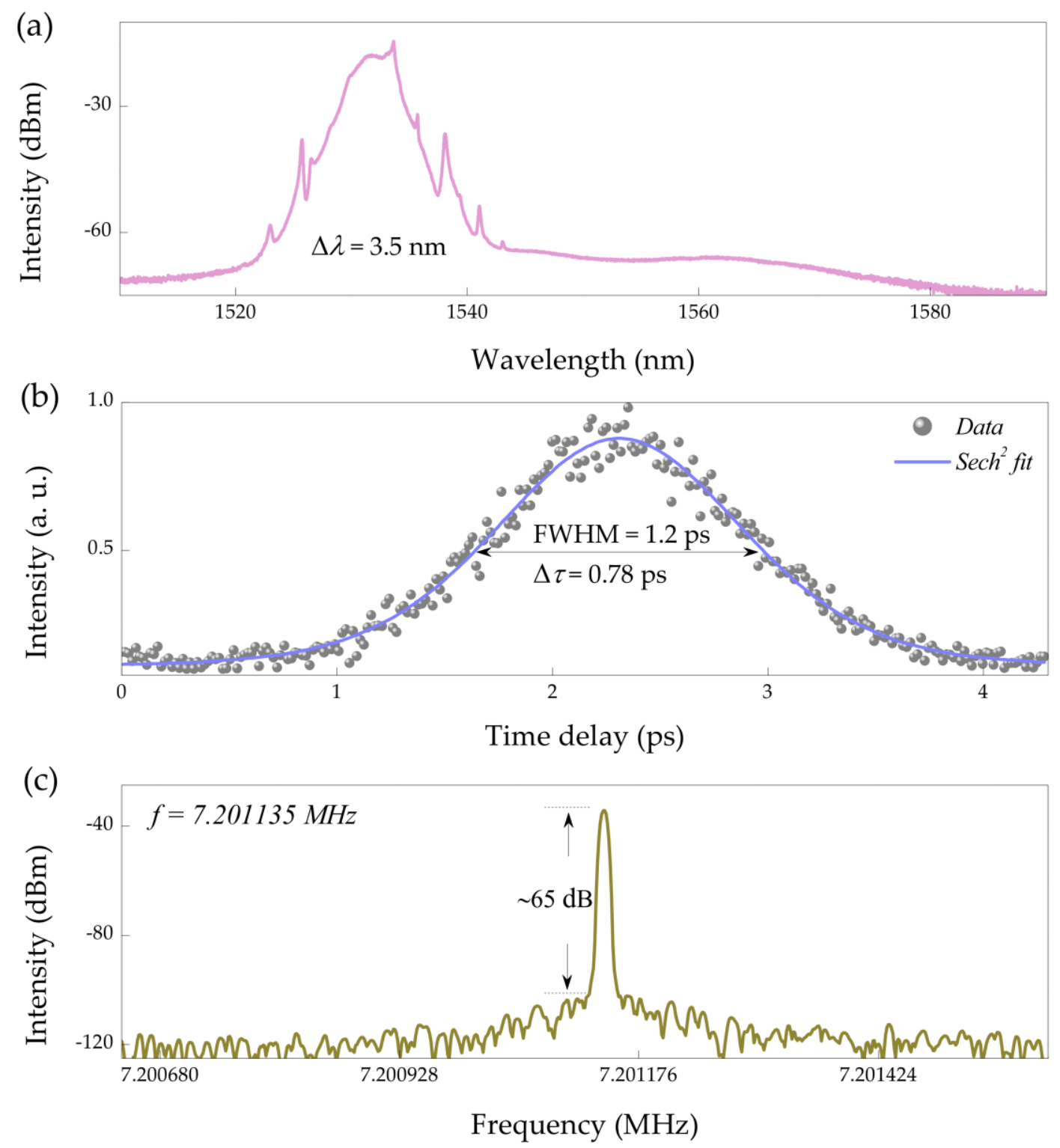

Figure 6. Single-wavelength conventional soliton. (a) Optical spectrum at $1532 \mathrm{~nm}$, (b) autocorrelation trace, and (c) corresponding fundamental radio-frequency spectrum.

\section{Conclusions}

A passively mode-locked dual-wavelength Er-doped fiber laser is demonstrated with a fiber-based PbS QDs SA. Compared with other nanomaterials, PbS QDs prepared by a modified hot-injection method have the advantages of fast relaxation time, wide bandwidth, large modulation depth, and thermal damage. Based on this PbS QDs SA, the pulse laser can operate in a dual-wavelength conventional solitons region centered at 1532 and $1559 \mathrm{~nm}$ with $3 \mathrm{~dB}$ bandwidths of 2.8 and $2.5 \mathrm{~nm}$, respectively. The experimental results reveal that our PbS QDs can be adopted as a broadband SA for application in pulse lasers.

Author Contributions: Conceptualization W.Z.; writing-original draft preparation, L.Y.; writingreview and editing, L.Y. and W.Z. All authors have read and agreed to the published version of the manuscript.

Funding: This research was funded by National Natural Science Foundation of China, grant number 61905118; Nanjing University of Posts and Telecommunications, grant number NY218023; and Research Center of Optical Communications Engineering \& Technology, Jiangsu Province, grant number ZXF201905. 
Institutional Review Board Statement: Not applicable.

Informed Consent Statement: Not applicable.

Data Availability Statement: Not applicable.

Conflicts of Interest: The authors declare no conflict of interest.

\section{References}

1. Martinez, A.; Sun, Z. Nanotube and graphene saturable absorbers for fibre lasers. Nat. Photon. 2013, 7, 842-845. [CrossRef]

2. Xu, N.; Wang, H.; Zhang, H.; Guo, L.; Shang, X.; Jiang, S.; Li, D. Palladium diselenide as a direct absorption saturable absorber for ultrafast mode-locked operations: From all anomalous dispersion to all normal dispersion. Nanophotonics 2020, 9, 4295-4306. [CrossRef]

3. Feng, J.; Li, X.; Shi, Z.; Zheng, C.; Li, X.; Leng, D.; Wang, Y.; Liu, J.; Zhu, L. 2D ductile transition metal chalcogenides (TMCs): A novel high-performance $\mathrm{Ag}_{2} \mathrm{~S}$ nanosheets for ultrafast photonics. Adv. Opt. Mater. 2019, 8, 1901762. [CrossRef]

4. Wu, X.; Yu, S.; Yang, H.; Li, W.; Liu, X.; Tong, L. Effective transfer of micron-size graphene to microfibers for photonic applications. Carbon 2016, 96, 1114-1119. [CrossRef]

5. Lee, E.; Choi, S.; Jeong, H.; Park, N.; Yim, W.; Kim, M.; Park, J.; Son, S.; Bae, S.; Kim, S.; et al. Active control of all-fibre graphene devices with electrical gating. Nat. Commu. 2015, 6, 6851. [CrossRef] [PubMed]

6. Wang, T.; Jin, X.; Yang, J.; Wu, J.; Yu, Q.; Pan, Z.; Shi, X.; Xu, Y.; Wu, H.; Wang, J.; et al. Oxidation-resistant black phosphorus enable highly ambient-stable ultrafast pulse generation at a $2 \mu \mathrm{m}$ Tm/Ho-doped fiber laser. ACS Appl. Mater. Interfaces 2019, 11, 36854-36862. [CrossRef] [PubMed]

7. Fu, B.; Sun, J.; Wang, C.; Shang, C.; Xu, L.; Li, J.; Zhang, H. MXenes: Synthesis, optical properties, and applications in ultrafast photonics. Small 2021, 17, 2006054. [CrossRef] [PubMed]

8. Song, Y.; Liang, Z.; Jiang, X.; Chen, Y.; Li, Z.; Lu, L.; Ge, Y.; Wang, K.; Zheng, J.; Lu, S.; et al. Few-layer antimonene decorated microfiber: Ultra-short pulse generation and all-optical thresholding with enhanced long term stability. 2D Mater. 2017, 4, 045010. [CrossRef]

9. Mao, D.; Du, B.; Yang, D.; Zhang, S.; Wang, Y.; Zhang, W.; She, X.; Cheng, H.; Zeng, H.; Zhao, J. Nonlinear saturable absorption of liquid-exfoliated molybdenum/tungsten ditelluride nanosheets. Small 2016, 12, 1489-1497. [CrossRef] [PubMed]

10. Luo, Z.; Liu, M.; Liu, H.; Zheng, X.; Luo, A.; Zhao, C.; Zhang, H.; Wen, S.; Xu, W. 2 GHz passively harmonic mode-locked fiber laser by a microfiber-based topological insulator saturable absorber. Opt. Lett. 2013, 38, 5212-5215. [CrossRef] [PubMed]

11. Wang, F.; Rozhin, A.; Scardaci, V.; Sun, Z.; Hennrich, F.; White, I.; Milne, W.; Ferrari, A. Wideband-tuneable, nanotube modelocked, fibre laser. Nat. Nanotechnol. 2008, 3, 738-742. [CrossRef]

12. Gladush, Y.; Mkrtchyan, A.; Kopylova, D.; Ivanenko, A.; Nyushkov, B.; Kobtsev, S.; Kokhanovskiy, A.; Khegai, A.; Melkumov, M.; Burdanova, M.; et al. Ionic liquid gated carbon nanotube saturable absorber for switchable pulse generation. Nano Lett. 2019, 19, 5836-5843. [CrossRef]

13. Rafailov, E.; Cataluna, M.; Sibbett, W. Mode-locked quantum-dot lasers. Nat. Photon. 2007, 1, 395-401. [CrossRef]

14. Guerreiro, P.; Ten, S.; Borrelli, N.; Butty, J.; Jabbour, G.; Peyghambarian, N. PbS quantum-dot doped glasses as saturable absorbers for mode locking of a Cr:Forsterite laser. Appl. Phys. Lett. 1997, 71, 1595-1597. [CrossRef]

15. Moreels, I.; Lambert, K.; Smeets, D. Size-dependent optical properties of colloidal PbS quantum dots. ACS Nano 2009, 3, 3023-3030. [CrossRef]

16. Lagatsky, A.; Malyarevich, A.; Savitski, V.; Gaponenko, M.; Yumashev, K.; Zhilin, A.; Brown, C.T. PbS quantum-dot-doped glass for efficient passive mode locking in a CW Yb: KYW laser. IEEE Photon. Techol. Lett. 2006, 18, 259-261. [CrossRef]

17. Lee, Y.; Chen, C.; Huang, C.; Chen, S.; Jiang, J. Passively Q-switched Er3+-doped fiber lasers using colloidal PbS quantum dot saturable absorber. Opt. Express 2016, 24, 10675-10681. [CrossRef] [PubMed]

18. Yun, L.; Qiu, Y.; Yang, C.; Xing, J.; Yu, K.; Xu, X.; Wei, W. PbS quantum dots as a saturable absorber for ultrafast laser. Photon. Res. 2018, 6, 1028-1032. [CrossRef]

19. Wundke, K.; Pötting, S.; Auxier, J.; Schülzgen, A.; Peyghambarian, N.; Borrelli, N. PbS quantum-dot-doped glasses for ultrashort pulse generation. Appl. Phys. Lett. 2000, 76, 10-12. [CrossRef]

20. Zhang, H.; Tang, D.; Wu, X.; Zhao, L. Multi-wavelength dissipative soliton operation of an erbium doped fiber laser. Opt. Express 2009, 17, 12692-12697. [CrossRef] [PubMed]

21. Liu, X.; Han, D.; Sun, Z.; Zeng, C.; Lu, H.; Mao, D.; Cui, Y.; Wang, F. Versatile multi-wavelength ultrafast fiber laser mode-locked by carbon nanotubes. Sci. Rep. 2013, 3, 2718. [CrossRef]

22. Wu, Z.; Fu, S.; Chen, C.; Tang, M.; Shum, P.; Liu, D. Dual-state dissipative solitons from an all-normal dispersion erbium-doped fiber laser: Continuous wavelength tuning and multi-wavelength emission. Opt. Lett. 2015, 40, 2684-2687. [CrossRef]

23. Zhao, C.; Dai, L.; Huang, Q.; Huang, Z.; Mou, C.; Araimi, M.; Rozhin, A.; Sergeyev, S.; Luo, Z. Dynamic polarization attractors of dissipative solitons from carbon nanotube mode-locked Er-doped laser. Nanophotonics 2020, 9, 2437-2443. [CrossRef]

24. Huang, S.; Wang, Y.; Yan, P.; Zhao, J.; Li, H.; Lin, R. Tunable and switchable multi-wavelength dissipative soliton generation in a graphene oxide mode-locked Yb-doped fiber laser. Opt. Express 2014, 22, 11417-11426. [CrossRef] [PubMed]

25. Yun, L. Black phosphorus saturable absorber for dual-wavelength polarization-locked vector soliton generation. Opt. Express 2017, 25, 32380-32385. [CrossRef] 
26. Zhang, Y.; Li, X.; Qyyum, A.; Feng, T.; Guo, P.; Jiang, J. Lead sulfide nanoparticles for dual-wavelength ultrashort pulse generation. Nanotechnology 2020, 31, 085202. [CrossRef]

27. Hines, M.; Scholes, G. Colloidal PbS nanocrystals with size-tunable near-infrared emission: Observation of post-synthesis self-narrowing of the particle size distribution. Adv. Mater. 2003, 15, 1844-1849. [CrossRef]

28. Liu, X.; Yang, H.; Cui, Y.; Chen, G.; Yang, Y.; Wu, X.; Yao, X.; Han, D.; Han, X.; Zeng, C.; et al. Graphene-clad microfibre saturable absorber for ultrafast fibre lasers. Sci. Rep. 2016, 6, 26024. [CrossRef]

29. Shang, C.; Zhang, Y.; Qin, H.; He, B.; Zhang, C.; Sun, J.; Li, J.; Ma, J.; Ji, X.; Xu, L.; et al. Review on wavelength-tunable pulsed fiber lasers based on 2D materials. Opt. Laser Technol. 2020, 131, 106375. [CrossRef]

30. Agrawal, G. Nonlinear Fiber Optics, 4th ed.; Academic Press: Cambridge, MA, USA, 2007. 